\title{
Steering effect on the shape of islands for homoepitaxial growth of $\mathrm{Cu}$ on $\mathrm{Cu}(001)$
}

\author{
Jikeun Seo, ${ }^{1}$ S.-M. Kwon, ${ }^{2}$ H.-Y. Kim, ${ }^{2}$ and J.-S. Kim ${ }^{2}$ \\ ${ }^{1}$ Division of General Education, Chodang University, Muan 534-701, Republic of Korea \\ ${ }^{2}$ Department of Physics, Sook-Myung Women's University, Seoul 140-742, Republic of Korea
}

(Dated: October 26, 2018)

\begin{abstract}
The steering effect on the growth of islands is investigated by combining molecular dynamics (MD) and kinetic Monte Carlo (KMC) simulations. Dynamics of depositing atoms and kinetics of atoms on a substrate are realized by MD and KMC, respectively. The reported experimental results on the asymmetric island growth [van Dijken et al., Phys. Rev. Lett. 82, 4038 (1999).] is well reproduced. A salient phenomenon, the reversal of the asymmetry, is found as the island size increases, and attributed to the asymmetric flux on the lower terrace of island.
\end{abstract}

PACS numbers: PACS numbers: 68.35.-p, 68.37.-d 
The growth of thin film is an essential step for many modern technologies and scientific investigation, and a lot of efforts have been made to understand and tailor the growth process. There have been many studies on the effect of energetic [1] and kinetic [2] variables to the growth of thin film, while minor attention has been paid to the role of dynamic variables such as deposition conditions. Recently, van Dijken et al.[3] reported the growth of rectangular $\mathrm{Cu}$ islands on square-symmetric $\mathrm{Cu}(001)$ when the deposition was made at grazing incidence angle. Furthermore, they controlled the shape of $\mathrm{Co}$ island on $\mathrm{Cu}(001)$ by varying the deposition angle and successfully manipulated its magnetic anisotropy [4. Their work clearly reveals the importance of dynamic variable and expand the adjustable parameters for the growth of thin film.

An atomistic picture for the asymmetric island growth was also proposed by van Dijken et al.[3]; Incident atoms see a modified potential by pre-existing island and as a result the incoming flux is focused on the upper terrace of the island near the front edge, while depleted on the lower terrace near the rear edge of the island. Such steering effect is argued to result in the shortened edge length along the deposition direction, $x$-axis, and leaves the edge perpendicular to the deposition direction, $y$-axis, relatively longer. The model, however, is based on a qualitative argument without any detailed kinetic description on island growth from the inhomogeneous flux distribution, and the steering effect is considered only in the incident plane rather than in the three dimensional space as it should be. Independently, Zhong et al. [5] proposed that the enhanced corner crossing diffusion at the front edge due to the transient mobility of the deposited atom increased the growth speed along the $y$-direction relative to that along the $x$-direction. However, the very existence and the role of the transient mobility still remains controversial [6]. Steering effect has also been studied in the roughening in amorphous thin film growth by MD[7] and non-linear stochastic equation [8]. For the growth of a crystalline film, only recently Montalenti and Voter [9] reported the steering induced instability of $\mathrm{Ag}$ film on $\mathrm{Ag}(001)$, but the study was restricted to the roughening of the film on a substrate at $0 \mathrm{~K}$ and to the deposition at normal incidence.

The purpose of the present study is to investigate the steering effect on the growth of asymmetric islands by performing a realistic simulation combining KMC and MD simulation; MD simulation is executed to calculate the trajectory of depositing atoms in three dimensional space, only when a deposition event is selected in KMC. Before the next deposition event is selected, various diffusion events of adatoms are realized by KMC simulation [10]. 
In the present study, the asymmetric island shape observed in the experimental study[3] is well reproduced. Moreover, a salient phenomenon, the reversal of the asymmetry of island from elongation in the $y$-direction to the $x$-direction, is found as the coverage or the substrate temperature increases. The asymmetric shape of the island is attributed mainly to the asymmetric deposition flux on the lower terrace of island that in turn depends on its size.

In MD simulation, Lennard-Jones potential in the form of $U(r)=4 D\left[(\sigma / r)^{12}-(\sigma / r)^{6}\right]$ with $D=0.4093 \mathrm{eV}$ and $\sigma=2.338 \AA$ is used for the interaction between the incident atom and surface atoms [6], and Verlet algorithm is adapted. The simulation box is composed of 6 layer high empty space in $z$-direction on an fcc(001) surface of $400 \times 400$ atoms with periodic boundary condition in the plane. To reduce computational efforts, during deposition, the atoms on the substrate are frozen at each lattice site. (The surface lattice constant, $a_{0}=2.56$ $\AA$ and the interlayer spacing, $d=1.805 \AA$ ) The incident atom along [110] direction has initial kinetic energy of $0.15 \mathrm{eV}$ determined from the melting temperature of $\mathrm{Cu}$ and follows the trajectory determined from the interaction potential starting at the initial height of $10 a_{0}$ until the atom experiences the repulsive force by pre-existing atoms on the substrate. In the present simulation, no transient mobility is taken into account.

For the diffusion processes, 12 different ones are taken into account including those suggested by Furman et al. [1], and their diffusion barriers are adopted. Some of the most influential diffusion barriers are listed in Table 1.

From the simulated morphology, the mound radius, which can directly be compared with the diffraction results [3], is determined as the first zero of the height-height correlation function $<h(\mathbf{r}) h(0)>-<h>^{2}$. To increase the statistical reliability, simulations are performed 60 times under the same growth condition, and the mound radius is calculated over the whole set of simulated morphologies.

In Fig. 1 we summarize the simulation results for the growth of $\mathrm{Cu}$ atoms on $\mathrm{Cu}(001)$ under the identical growth conditions to those of the previous experiment [3]. (We denote the mound radius along the $x$-axis by $L$ and that along the $y$-axis by $W$.) For the normal deposition, $W$ and $L$ are identical at all coverages, while for the deposition at an angle, $80^{\circ}$ off the surface normal, the symmetry is broken and the difference between $W$ and $L$ becomes larger as the coverage increases. At 0.5 monolayer $(\mathrm{ML}), W$ is larger than $\mathrm{L}$ by $5 \%$ reproducing the previously reported result [3]. It is noteworthy that we reproduce the 
experimental result [3] without taking any transient mobility into account. The inset of Fig. 1 shows that for constant coverage of $0.5 \mathrm{ML}$, the aspect ratio, $W / L$, increases, as does the deposition angle. This result demonstrates that the asymmetry of the island shape is closely related to the reduced symmetry of the deposition geometry.

The top figure in Fig. 2(a) reproduces the flux distribution along the $x$-axis through the center of a pre-existing island as reported in Ref. 3, which herein is called the front and rear edge $(F R$-edge $)$ steering effect. Also found in the present study, as one may see in the following figures of Fig. 2(a), is the strong dependence of the flux distribution on the island size; as the island size becomes small, the portion of the island with enhanced flux is enlarged. Besides, in Fig. 2(b), an additional steering effect called herein the side edge $(S$-edge $)$ steering is observed; the trajectories of incident atoms are curved toward both side edges and enhanced flux is found along both side edges. As will be discussed later, both the dependence of deposition flux on the island size and the relative strength of FR-edge steering to $S$-edge steering have great impact on shaping the island.

To have a comprehensive picture on the asymmetric island growth, a series of simulation is performed with various substrate temperatures. Fig. 3 shows the dependence of $L$ and $W$ on coverage at various growth temperatures and the inset summarizes the temperature dependence of the aspect ratio at 0.5 ML. A stunning new phenomenon, the reversal of the asymmetry of island around $230 \mathrm{~K}$, is found; $W>L$ above $230 \mathrm{~K}$ as observed in Ref. 3 , but $W<L$ below 230K. Such a reversal of the island shape has never been predicted before.

We find that aforementioned, apparently diverse island growth can be understood by exploring the nature of the inhomogeneous deposition flux. The inhomogeneous flux is expected to manifest itself in two different ways; 1) asymmetric downhill current from the top of the island and 2) asymmetric deposition flux around the lower terrace of the island. As for the first one, despite the inhomogeneous deposition flux on the upper terrace of the island as shown in Fig. 2, the ratio of the downhill current per unit edge length over $x$-directional edge to that over $y$-directional edges is very close to 1.0 when we count the atoms coming over the ES barrier during growth simulation. This direction-independence of the downhill diffusion current occurs because the terrace diffusion on top of the island is more frequent by $\sim 10^{2}$ times than the downhill diffusion over ES barrier, and effectively homogenizes the inhomogeneous deposition flux on the island before atoms come down over the barrier. 
As for the second one, we investigate the flux distribution on the lower terrace of the island. In Fig. 4(a), shown is the average deposition flux on terraces within $3 a_{0}$ from the side edges (S-terrace) and that from the front and rear edges (FR-terrace), respectively, as a function of the island size. The flux on the FR-terrace decreases monotonically, but that on the S-terrace shows a maximum as the island becomes larger. For the island with its edge length smaller than $10 a_{0}$, the flux on FR-terrace is larger than that on S-terrace, but the other way is true for the larger island.

Aforementioned dependence of the deposition flux on the island size can be understood from the flux distribution shown in Figs. 2 and 5. For small islands(Fig. 2(a) middle and bottom), the perturbed potentials at front and rear edges interfere and result in the increased flux on the FR-terrace. As the island size increases, the flux near the rear edge becomes relatively small (Fig. 2(a) top) due to the uninterfered rear edge potential. Hence, the average flux on the FR-terrace monotonically decreases as the island size increases as found in Fig. 4(a).

On the other hand, the average flux on the S-terrace is influenced by two counter-acting factors; firstly, the flux on the S-terrace increases with the island size, because the longer the edges the more atoms moving parallel to the side edge are attracted to the edge. However, the flux on S-terrace is not homogeneous along the side edge and another intricate variable is involved (Fig. 5). For small islands (Fig. 5 top and middle), the deposition flux is enhanced over the whole edge. As the island size increases, the relative portion of the side edge with pronounced flux enhancement gradually decreases(Fig. 5 bottom). This suggests that the average flux over the side edge will gradually decrease as the edge length increases. Hence, the competition between these two factors results in the maximum of the average flux at the island size of 17-20 $a_{0}$, explaining the dependence of the average flux on the island size observed in Fig. 4(a). The origin of the inhomogeneous enhancement of flux along the side edges on S-terraces can be found by the comparison of Figs. 2(a) and 5. The flux profile along the side edge(Fig. 5) shows quite a resemblance with that along the deposition direction through the center of the island (Fig. 2(a)). It suggests that the side edge flux is determined not solely by the $S$-edge steering but cooperatively by the FR-edge steering.

The island-size dependence of the flux ratio as shown in Fig. 4(b) is quite similar to the temperature dependence of the aspect ratio of the islands as shown in the inset of Fig. 3. Keeping in mind that the mean island size monotonically increases with the substrate 
temperature for the same coverage, it suggests that the temperature dependence of the aspect ratio of the island may originate from the dependence of the flux ratio on the island size; At low substrate temperature where small islands form, the average flux on FR-terrace is larger than that on S-terrace and results in asymmetric island having $L>W$, and vice versa. We examine this idea further by plotting the aspect ratio of the islands as a function of the mean size of islands formed with varying growth conditions. As shown in Fig. 4(b), even though the data are collected from uncorrelated simulations, there exists a strong correlation between the aspect ratio and the island size. Furthermore, it reproduces all the features found in the dependence of the flux ratio on the island size, especially the crossover (aspect ratio $=1$ ), maximum, and thereafter monotonic decrease of the ratio. (The island sizes where the crossover and the maximum in the aspect ratio occur are larger than the corresponding ones in the flux ratio. This is understood from the fact that the island size is determined by the integration of deposition flux.) It suggests that the most important factor determining the shape of the island is the deposition flux on the lower terrace of the island, which in turn is determined by the size of the island. If that conjecture is correct, the asymmetric island shape $(L>W)$ should also be observed in the initial deposition stage and so does the reversal of the asymmetry with further growth unless the temperature is too low to form islands larger than the crossover size, because the island size is always small at the early stage of the island growth, irrespective of the substrate temperature. As indicated with + signs in Fig. 3, the reversal of the asymmetry is indeed observed at similar island sizes, regardless of the substrate temperatures above $230 \mathrm{~K}$, reassuring that the island-size dependent flux ratio at the lower terrace of the island is the critical factor for shaping the island.

In summary, the present simulation combining MD and KMC properly reproduces the previously reported rectangular island growth of $\mathrm{Cu} / \mathrm{Cu}(001)$ under deposition at a grazing incidence angle, and shows that the transient mobility is not a necessary condition for the asymmetric island growth. Instead, the asymmetry of the island shape is attributed mainly to the asymmetry of the deposition flux on the lower terrace of islands. Also found is the existence of the reversal of the aspect ratio of asymmetric islands, depending on the substrate temperature or the island size. This, so far, unanticipated phenomenon remains to be proven by further experimental study. It is also found important that, for a proper interpretation of the experimental results, the steering effect should be treated in full three 
dimension.

This work is support by KISTEP(00-B-WB-07-A-01) (JK).

[1] E. Bauer, in The Chemical Physics of Solid Surfaces and Heterogeneous Catalysis Vol. 3B, edited by D. A. King and D. P. Woodruff (Elsevier, Amsterdam, 1984).

[2] H. Brune, Surf. Sci. Rep. 31, 121 (1998); The Chemical Physics of Solid Surfaces Vol. 8, edited by D. A. King and D. P. Woodruff (Elsevier, Amsterdam, 1997); Morphological Organization in Expitaxial Growth and Removal, edited by Z. Zhang and M. G. lagally (World Scientific, Singapore, 1998).

[3] S. V. Dijken, L. C. Jorritsma, and B. Poelsema, Phys. Rev. Lett. 82, 4038 (1999); S. V. Dijken, L. C. Jorritsma, and B. Poelsema, Phys. Rev. B 61, 14047 (2000).

[4] S. van Dijken, G. D. Santo, and B. Poelsema, Appl. Phys. Lett. 77, 2030 (2000).

[5] J. Zhong, E. Wang, Q. Niu, and Z. Zhang, Phys. Rev. Lett. 84, 3895 (2000).

[6] D. E. Sanders and A. E. DePristo, Surf. Sci. 254, 341 (1991).

[7] W. D. Luedtke and U. Landman, Phys. Rev. B 40, 11733 (1989).

[8] M. Raible, S. J. Linz, and P. Hänggi, Phys. Rev. E 62, 1691 (2000).

[9] F. Montalenti and A. F. Voter, Phys. Rev. B 64, R081401 (2001).

[10] Similar approach is found in the following article; S.W. Levine, J. R. Engstrom, and P. Clancy, Surf. Sci. 401, 112 (1998).

[11] I. Furman, O. Biham, Jiang-Kai Zuo, A. K. Swan, and John F. Wendelken, Phys. Rev. B 62, R10649 (2000). 
TABLE I: Some of the diffusion barriers and diffusion parameters used in simulation. Notations in the bracket [ ] are from Furman et al. [11].

\begin{tabular}{cc}
\hline \hline type of diffusion & diffusion barrier \\
\hline free jump $\left[E_{0}\right]$ & $0.485 \mathrm{eV}$ \\
dimer lateral bond break $\left[E_{2}\right]$ & $0.463 \mathrm{eV}$ \\
re-estabilishing NN bond $\left[E_{4}\right]$ & $0.183 \mathrm{eV}$ \\
Erlich-Schwöbel $(\mathrm{ES})$ barrier $\left(\Delta E_{S}\right)$ & $0.1 \mathrm{eV}$ \\
\hline jump frequency $\left(\nu_{0}\right)$ & $2.4 \times 10^{13}$ \\
deposition rate $\left(F_{0}\right)$ & $0.00416 \mathrm{ML} / \mathrm{s}$ \\
\hline \hline
\end{tabular}

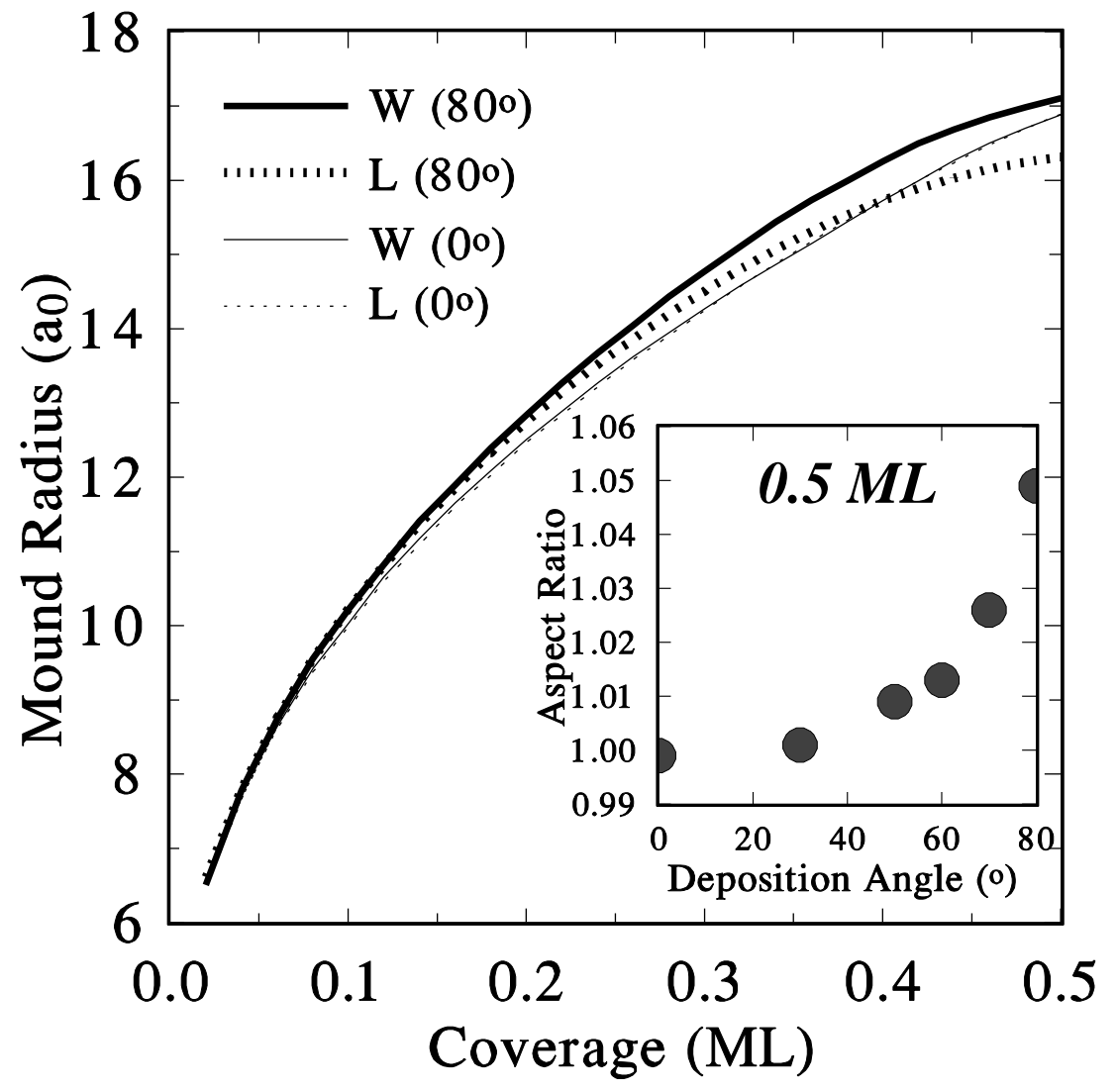

FIG. 1: Mound radius along both $\mathrm{x}$ and $\mathrm{y}$ directions for $\mathrm{Cu}$ islands grown on $\mathrm{Cu}(001)$ at $250 \mathrm{~K}$. Inset : Aspect ratio of mound radii, $W / L$, versus deposition angle (from the surface normal). 


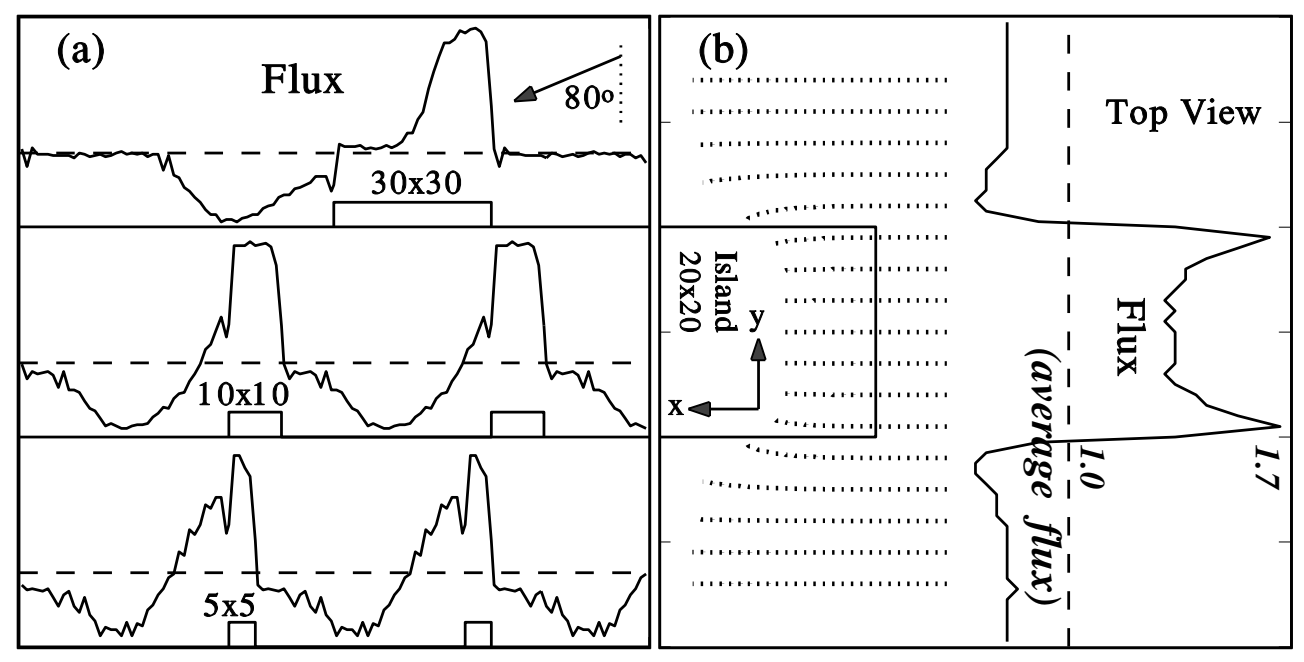

FIG. 2: The deposition flux and atomic trajectory calculated from MD simulation at deposition angle of 80 degree. (a) Deposition flux along x direction for islands of sizes, $30 a_{0}$ (top), 10 $a_{0}$ (middle), and $5 a_{0}$ (bottom), respectively. (b)Top view of the trajectory of incident atoms near a pre-existing island, and flux distribution across the deposition direction, y direction. Long dashed line represents the normalized flux. 


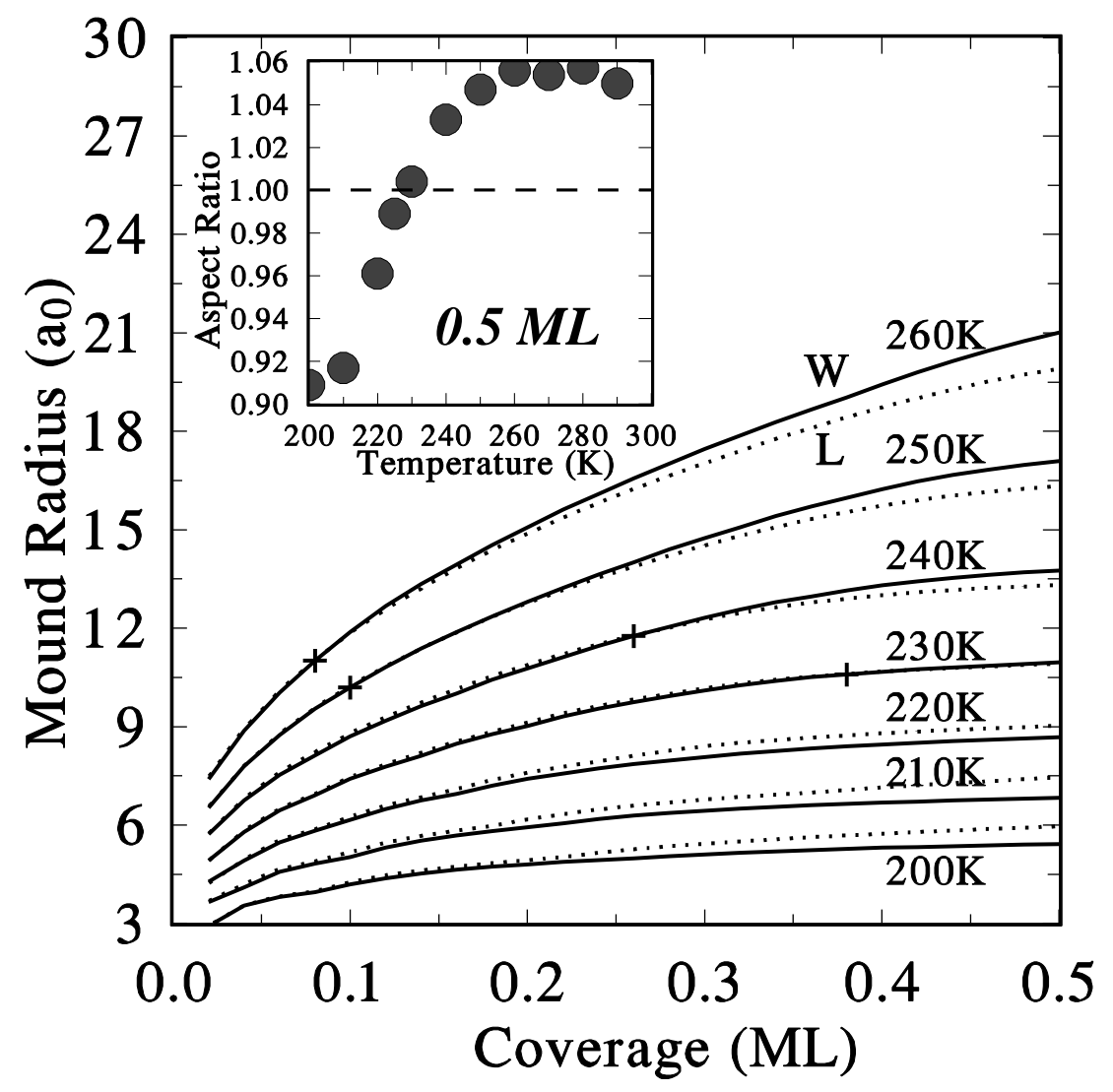

FIG. 3: Temperature dependence of mound radius. Solid curves represent mound radius $(W)$ along $y$-direction and dotted curves correspond to that $(L)$ along $x$-direction. Inset : Aspect ratio $(W / L)$ for $0.5 \mathrm{ML}$ growth. 


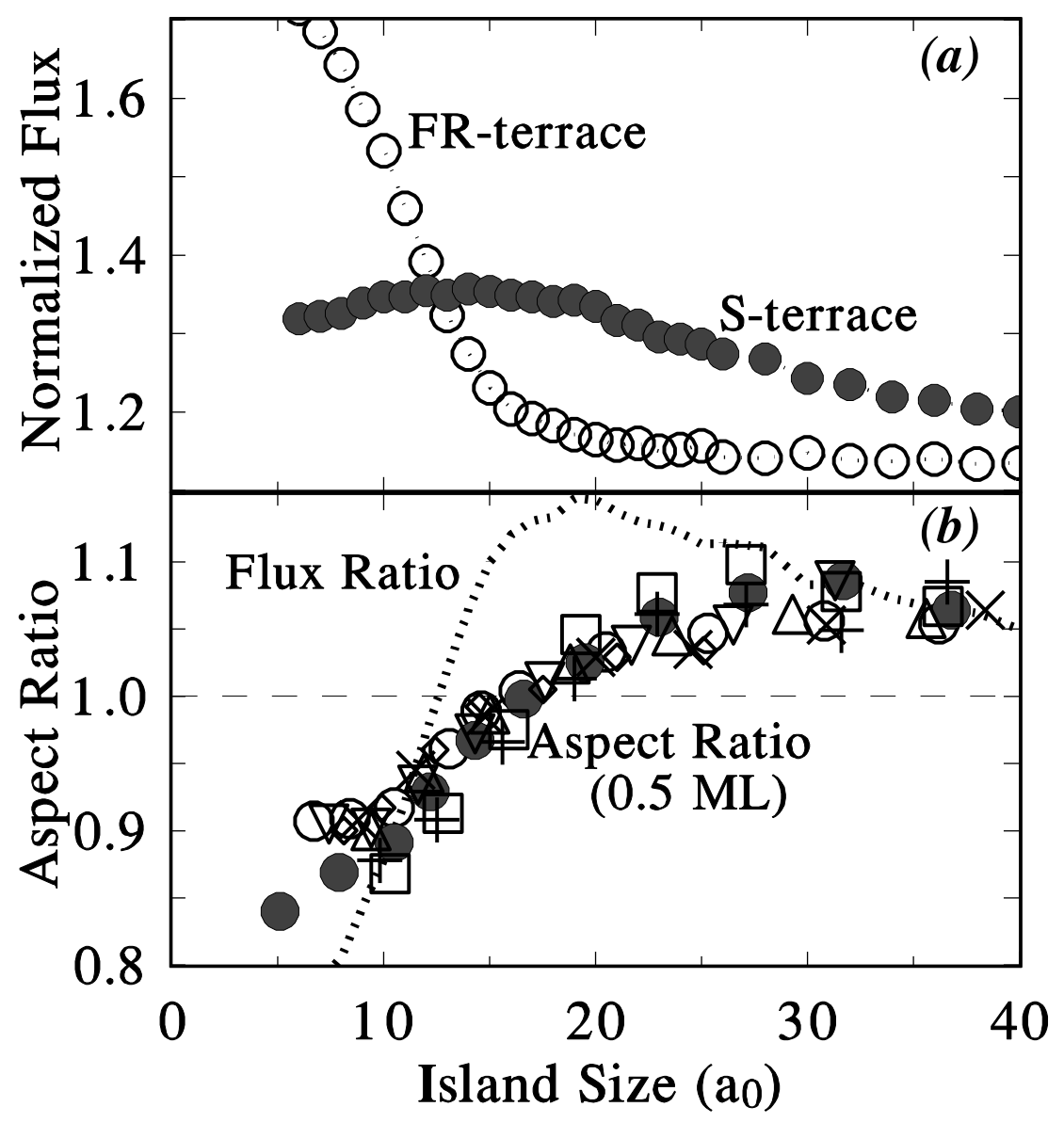

FIG. 4: Deposition flux and the aspect ratio according to the island size. (a)The filled and open circles represent the average deposition flux on $S$-terrace $\left(F_{\mathrm{S} \text {-terrace }}\right)$ and that on $F R$ terrace $\left(F_{\mathrm{FR} \text {-terrace }}\right)$, respectively. Each flux is normalized to the mean flux. (b)The ratio of deposition fluxes, $\left(F_{\mathrm{S}-\text { terrace }} / F_{\mathrm{FR} \text {-terrace }}\right.$; dotted curve) and the aspect ratio $(W / L)$ for $0.5 \mathrm{ML}$ film grown with various temperatures $(190 \mathrm{~K}-300 \mathrm{~K})$ and diffusion parameters (open circle(Table I), $+\left(\Delta E_{S c h}=0.05 \mathrm{eV}\right), \operatorname{box}\left(\Delta E_{S c h}=0.00 \mathrm{eV}\right), \operatorname{diamond}\left(\Delta E_{S c h}=0.15 \mathrm{eV}\right), \operatorname{closed} \operatorname{circle}\left(E_{2}=0.553\right.$ $\left.\mathrm{eV}, E_{4}=0.485 \mathrm{eV}\right), \Delta\left(\right.$ deposition rate $\left.1 / 2 F_{0}\right), \mathrm{X}\left(\right.$ deposition rate $\left.\left.1 / 12 F_{0}\right), \nabla\left(\nu_{0}=1.2 \times 10^{13}\right)\right)$ are plotted as a function of the island size. 


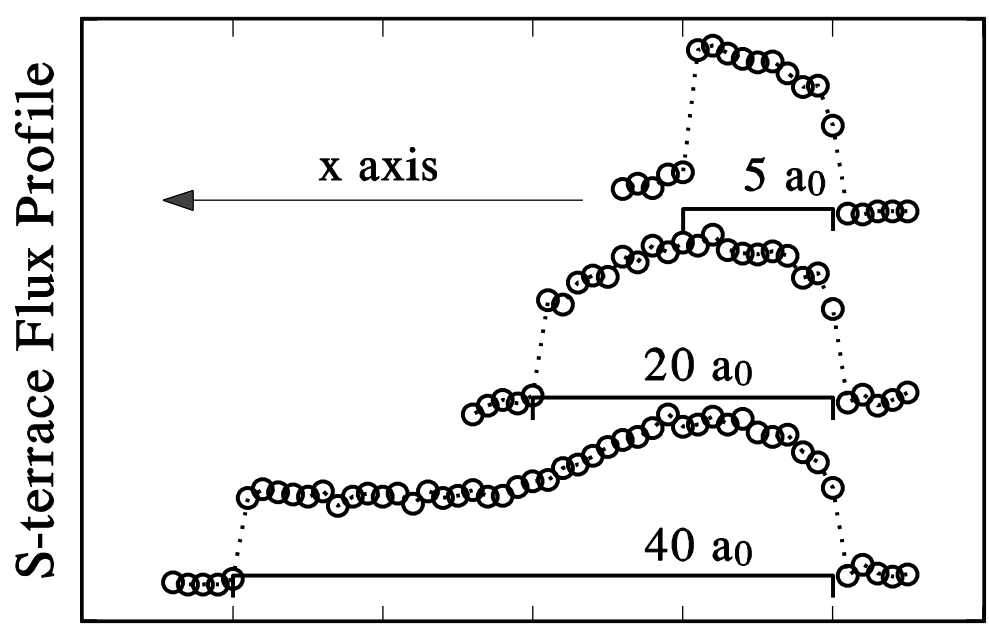

FIG. 5: Flux profile on S-terrace along the side edge for islands of varying sizes, 5, 20, and $40 a_{0}$ long. Solid lines represent both the position of pre-existing islands and mean flux. 